\title{
The Electron Spin as Resulting From the Ether Elasticity
}

\author{
David Zareski ${ }^{1}$ \\ ${ }^{1}$ I.A.I, Israel Aerospace Industries Yehud, Israel \\ Correspondence: David Zareski, Shilo Str. 74, Roch HaAyin, Israel. E-mail: zareski@inter.net.il
}

Received: July 18, $2014 \quad$ Accepted: August 12, $2014 \quad$ Online Published: August 14, 2014
doi:10.5539/apr.v6n5p41
URL: http://dx.doi.org/10.5539/apr.v6n5p41

\begin{abstract}
We recall how the Maxwell equations ensue from the Navier-Stokes-Durand equation of elasticity that governs the field of the displacements $\xi$ of the points of the ether shown to be an elastic medium. Such a field $\xi$ can be constituted by waves propagated in this ether. In previous papers we have generalized the waves $\xi$ associated to photons to waves $\xi(\mathrm{m}, \mathrm{e})$ associated to $\operatorname{Par}(\mathrm{m}, \mathrm{e}) \mathrm{s}$, (i.e., particles of mass $\mathrm{m}$ and electrical charges e), and demonstrated that a moving $\operatorname{Par}(\mathrm{m}, \mathrm{e})$, as, e.g., an electron is a superposition $\hat{\xi}(\mathrm{m}, \mathrm{e})$ of waves $\xi(\mathrm{m}, \mathrm{e})$ that forms a small globule moving with the velocity $\mathbf{V}$ of this $\operatorname{Par}(\mathrm{m}, \mathrm{e})$. That is to say that a moving particle is a moving small globule $\hat{\xi}(\mathrm{m}, \mathrm{e})$ of ether deformation. Now in its motion, $\hat{\xi}(\mathrm{m}, \mathrm{e})$ creates out side of it, a field $\xi \hat{\xi}(\mathrm{m}, \mathrm{e})$ of ether deformation from witch ensues the electromagnetic field created by $\hat{\xi}(\mathrm{m}, \mathrm{e})$. The induced field $\xi \hat{\xi}(\mathrm{m}, \mathrm{e})]$ being denoted without ambiguity also simply by $\xi$, it appears, as shown here below, that the magnetic field $\mathbf{H}$ ensuing from $\xi$, is the field of the local velocities $\partial_{t} \xi$ of the points of the ether, i.e., $\mathbf{H}=\partial_{t} \xi$. The fundamental fact that we demonstrate, is that on a fixed observatory point $\mathbf{r}_{\mathrm{ob}}$ near at a given instant to the moving electron, i.e., to the moving $\hat{\xi}(\mathrm{m}, \mathrm{e})$, the velocity of the ether denoted there by $\partial_{\mathrm{t}} \xi_{\mathrm{ob}}$ is of the same form as the velocity of a point of a rotating solid. This phenomenon is the spin of the electron, that, in a quantum state of an atom can take only quantized values.
\end{abstract}

Résumé. Nous rappelons comment les équations de Maxwell découlent de l'équation de Navier-Stokes-Durand de l'élasticité qui régis le champ des déplacements $\xi$ des points de l'éther montré être un milieu élastique. Un tel champ $\xi$ peut être constitué d'ondes se propageant dans l'éther. Dans des articles antérieurs nous avons généralisé les ondes $\xi$ associées aux photons en les ondes $\xi(\mathrm{m}, \mathrm{e})$ associées aux $\operatorname{Par}(\mathrm{m}, \mathrm{e}) \mathrm{s}$, (i.e., particules de masse $\mathrm{m}$ et de charge électrique e $)$, et démontré qu'une $\operatorname{Par}(\mathrm{m}, \mathrm{e})$ mobile, comme par exemple un électron est une superposition $\hat{\xi}(\mathrm{m}, \mathrm{e})$ d'ondes $\xi(\mathrm{m}, \mathrm{e})$ formant un petit globule mouvant a la vitesse $\mathbf{V}$ de cette $\operatorname{Par}(\mathrm{m}, \mathrm{e})$. C'est-à-dire qu'une particule mobile est petit globule $\hat{\xi}(\mathrm{m}, \mathrm{e})$ mobile de déformation de l'éther. Or, dans son mouvement, $\hat{\xi}(\mathrm{m}, \mathrm{e})$ crée en dehors de lui, un champ $\xi \hat{\xi}[\mathrm{m}, \mathrm{e})]$ de déformation de l'éther d'où s'ensuit le champ électromagnétique créé par $\hat{\xi}(\mathrm{m}, \mathrm{e})$. Ce champ induit $\hat{\xi} \hat{\xi}(\mathrm{m}, \mathrm{e})]$ étant dénoté sans ambigüité simplement $\xi$, il apparait, comme il est montre ci-dessous, que le champ magnétique $\mathbf{H}$ qui découle de $\boldsymbol{\xi}$, est le champ des vitesses locales $\partial_{t} \xi$ des points de l'éther, i.e., $\mathbf{H}=\partial_{t} \xi$. Le fait fondamental que nous démontrons, est que en un point d'observation $\mathbf{r}_{\mathrm{ob}}$ proche, a un instant donné, l'électron mobile, i.e., de $\hat{\xi}(\mathrm{m}, \mathrm{e})$, la vitesse de l'éther, dénotée $\partial_{\mathrm{t}} \xi_{\mathrm{ob}}$, est de la même forme que la vitesse d'un point d'un solide en rotation. Ce phénomène est le spin de l'électron, qui, dans un état quantique d'un atome ne peut prendre que des valeurs quantifiées.

Keywords: ether elasticity theory, physical signification of the spin of the mobile electron

\section{Introduction}

Maxwell and Einstein presumed the existence of an ether, Cf. citations from Maxwell (1954) and Einstein (1920), rewritten, e.g., in Zareski (2013). In this context, we showed in Zareski $(2001,2012)$ and also recalled here below, that the Maxwell equations of electromagnetism ensue from the case of elasticity theory where the elastic medium is the ether, denoted $\mathbb{E}$, on which acts a volumetric density $\mathbf{C}$ of couples of forces that create the field $\xi$ of the displacements of the points of $\mathrm{E}$. This field $\xi$ is governed by the Navier-Stokes-Durand equation, rewritten in Equation (1) here below, that generates these Maxwell equations.

These results stimulated us to investigate the physical common nature of the fields and of the particles that will suppress the following contradiction and inconsistency cited by Einstein in Einstein (1991) in which he wrote in particular: 
.....the combination of the idea of a continuous field with the conception of a material point discontinuous in space appears inconsistent.....

To this purpose, it was natural to extend this elastic interpretation to the case where the particles are not only photons, but can also be $\operatorname{Par}(\mathrm{m}, \mathrm{e})$, i.e., a particle of mass $\mathrm{m}$ and of electrical charge e, that can be submitted to incident electromagnetic and or gravitational fields. This extension was achieved, in Zareski $(2012,2013)$ where we showed that the Lagrange-Einstein function $\mathrm{L}_{\mathrm{G}}$ of such a $\operatorname{Par}(\mathrm{m}, \mathrm{e})$ do not only yields the particle four-motion equation, but also is such that $\phi$, defined by $\hbar \mathrm{d} \phi / \mathrm{dt}=\mathrm{L}_{\mathrm{G}}$, is the phase of a wave associated to these particles. Thus $\mathrm{L}_{\mathrm{G}}$ implies the existence of waves, of phase $\phi$, denoted $\xi(\mathrm{m}, \mathrm{e})$, associated to these particles, that generalize the wave $\xi(0,0)$ denoted simply $\xi$.

Now let us consider more deeply these $\xi(\mathrm{m}, \mathrm{e})$. Since, in general, waves are propagated in an elastic medium, it follows that like the waves $\xi$, the waves $\xi(\mathrm{m}, \mathrm{e})$ are propagated in $\mathrm{E}$. Since when $\mathrm{m}$ tends toward zero a $\xi(\mathrm{m}, \mathrm{e})$ becomes a $\xi$ which is a field of the displacements of the points of $\mathbb{E}$, if follows that a $\xi(\mathrm{m}, \mathrm{e})$ is, like a $\xi$, a field of the displacements of the points of $\mathrm{E}$. Since the waves $\xi$ are the solution of this Navier-Stokes-Durand equation (1), it follows, that the waves $\xi(\mathrm{m}, \mathrm{e})$ are solutions of an equation that generalizes Equation (1), where now the particle trajectory is a ray of such a wave. Therefore the particle trajectory equation obtained from the Einstein particle motion equation generalize the light ray equation. Furthermore, an important point is, Cf. Zareski (2013), is that:

A specific sum of $\xi(\mathrm{m}, \mathrm{e})$ waves forms a globule $\hat{\xi}(\mathrm{m}, \mathrm{e})$ that moves like the $\operatorname{Par}(\mathrm{m}, \mathrm{e})$ and contains all its parameters; reciprocally, a $\xi(\mathrm{m}, \mathrm{e})$ wave is a sum of such globules, $\hat{\xi}(\mathrm{m}, \mathrm{e})$, i.e., of particles.

In the present paper we are interested only to the fact that a $\operatorname{Par}(\mathrm{m}, \mathrm{e})$ is such a globule $\hat{\xi}(\mathrm{m}, \mathrm{e})$. In its motion this $\operatorname{Par}(\mathrm{m}, \mathrm{e})$, i.e., this $\hat{\xi}(\mathrm{m}, \mathrm{e})$ creates a Lienard-Wiechert covariant potential tensor from which one deduces the electromagnetic field and in particular the magnetic field $\mathbf{H}$.

Now, in the ether elasticity theory, this $\mathbf{H}$ is the velocity $\partial_{t} \xi$ of the ether points, Cf. Equation (3).

The fundamental fact that we demonstrate, is that on a fixed observatory point $\mathbf{r}_{\mathrm{ob}}$ near at a given instant to the moving electron, i.e., to the moving $\hat{\xi}(\mathrm{m}, \mathrm{e})$, the velocity of the ether denoted there by $\partial_{\mathrm{t}} \xi_{\mathrm{ob}}$ is of the same form as the velocity of a point of a rotating solid. This phenomenon is the electron spin which, as we show, in a quantum state of an atom, can take only quantized values.

\section{Notations and Recalls of "The Elastic Interpretation of Electromagnetism"}

The Greek, Resp. Latin indices take the values 1, 2, 3, 4, Resp. 1, 2, 3, these last refer to spatial quantities, while the index 4 refers to temporal quantities. $c$ denotes the light velocity in the "vacuum", $\partial_{t}$, Resp., $\partial_{t t}$, the partial time derivative $\partial / \partial \mathrm{t}$, Resp. $\partial^{2} /(\partial \mathrm{t})^{2}$, and $\dot{\mathrm{f}}$ the time derivative $\mathrm{df} / \mathrm{dt}$.

In Zareski (2001) we have developed the "elastic interpretation of electrodynamics". In this interpretation, the ether $\mathrm{E}$ is shown to be an elastic medium of which the field $\xi$ of the displacements of its points is governed by the Navier-Stokes-Durand equation

$$
\operatorname{curl}(\mathbf{C} / 2-\eta \operatorname{curl} \xi)=\rho \partial_{\mathrm{tt}} \xi,
$$

where $\mathbf{C}$ denotes the volumetric density of couples applied to $\mathrm{E}$, $\eta$, the elastic restoring rotation coefficient of $\mathrm{E}$, and $\rho$, the volumetric density of $\mathrm{E}$. By using the following variable changes:

$$
\begin{gathered}
\mathbf{E} \equiv \eta \operatorname{curl} \xi-\mathbf{C} / 2, \quad \mathbf{H} \equiv \partial_{\mathrm{t}} \xi, \quad \mathbf{B} \equiv \rho \partial_{\mathrm{t}} \xi, \\
\mathbf{J}_{\mathrm{e}} \equiv \partial_{\mathrm{t}} \mathbf{C} /(2 \eta), \quad \rho_{\mathrm{e}} \equiv-\operatorname{div}[\mathbf{C} /(2 \eta)],
\end{gathered}
$$

Equation (1) yields the four following equations:

$$
\begin{array}{rlrl}
\operatorname{curl} \mathbf{E}+\partial_{\mathrm{t}} \mathbf{B} & =0, & & \operatorname{curl} \mathbf{H}-\partial_{\mathrm{t}} \mathbf{E} / \eta=\mathbf{J}_{\mathrm{e}}, \\
\operatorname{div} \mathbf{B}=0, & & \operatorname{div} \mathbf{E}=\eta \rho_{\mathrm{e}} .
\end{array}
$$

that are the Maxwell equations. It ensues that:

Equation (2) represents the elastic interpretation of the electric field $\mathbf{E}$;

Equation (3) represents the elastic interpretation of the magnetic field $\mathbf{H}$;

$\rho$, that, in the elastic interpretation, is the volumetric density of the ether, is the coefficient of magnetic induction;

Equation (4) represents the elastic interpretation of the magnetic induction $\mathbf{B}$;

$\eta$ that, in the elastic interpretation, is the elastic restoring rotation coefficient of $\mathbb{E}$, is the inverse of the electric induction coefficient $\varepsilon_{0}$, i.e., $\eta \equiv 1 / \varepsilon_{0}$; 
$\partial_{\mathrm{t}} \mathbf{C} /(2 \eta)$ is the elastic interpretation of the volumetric density of electric currents $\mathbf{J}_{\mathrm{e}}$; and

$-\operatorname{div}[\mathbf{C} /(2 \eta)]$ is the elastic interpretation of the volumetric density of electric charges $\rho_{\mathrm{e}}$.

Furthermore, from (5) and (6) one deduces that $\mathbf{J}_{\mathrm{e}}$ and $\rho_{\mathrm{e}}$ verify the continuity equation

$$
\operatorname{div} \mathbf{J}_{\mathrm{e}}+\partial_{\mathrm{t}} \rho_{\mathrm{e}}=0
$$

that expresses the charge conservation.

One sees that like presumed by Maxwell and by Einstein, the ether elasticity theory leads to Maxwell's electromagnetism.

Note: Equation (1) was generalized in, e.g., Zareski (2013) by Equation (32).

\section{The Electron Spin as a Phenomenon Due to the Ether Elasticity}

At the observatory point $\mathbf{R}_{\mathrm{ob}}$, let consider the electromagnetic field created by a moving electric charge e of velocity $\mathbf{V}$. This field derives from the Lienard-Wiechert covariant potential tensor $A_{\mu}$ for which the expression is

$$
\mathrm{A}_{\mu}=-\frac{\mathrm{e}}{4 \pi \varepsilon_{0}} \frac{\mathrm{V}_{\mu}}{(\mathrm{Rc}-\mathbf{R} \cdot \mathbf{V})}
$$

where $V_{\mu}$ is such that $V_{4}=c$, and $V_{j}$ denote the covariant components of $\mathbf{V}$, and where $\mathbf{R}$ denotes the radius vector going from the center of the charge to the point of observation $\mathbf{R}_{\mathrm{ob}}$ at the retarded time. If $\mathbf{A}$ denotes the vector of covariant spatial components $A_{j}$, then the expression for the magnetic field $\mathbf{H}$ created by $\mathrm{e}$ is

$$
\mathbf{H} \equiv(\operatorname{curl} \mathbf{A}) / \rho .
$$

On account of Equation (12), (13), here above, and of Equations (63.8) and (63.9) of Landau and Lifshitz (1962) expressed in MKSA units, the explicit expression for $\mathbf{H}$ at $\mathbf{R}_{\mathrm{ob}}$, is

$$
\mathbf{H} \equiv \partial_{\mathrm{t}} \xi=\frac{\mathrm{e} \mathbf{R}}{4 \pi \mathrm{c} \rho \varepsilon_{0} \mathrm{R}(\mathrm{R}-\mathbf{R} \cdot \mathbf{V} / \mathrm{c})^{3}} \wedge\left\{\frac{(\mathbf{R}-\mathbf{V R} / \mathrm{c})}{\beta^{2}}+\frac{\mathbf{R} \wedge[(\mathbf{R}-\mathbf{V R} / \mathrm{c}) \wedge \dot{\mathbf{V}}]}{\mathrm{c}^{2}}\right\},
$$

where $\beta^{2} \equiv 1 /\left(1-\mathrm{V}^{2} / \mathrm{c}^{2}\right)$, and $\dot{\mathbf{V}} \equiv \mathrm{d} \mathbf{V} / \mathrm{dt}$ denotes the acceleration of e.

Let us consider now the case where $\mathbf{R}_{\mathrm{ob}}$ is very close to e and then denoted then by $\mathbf{r}_{\mathrm{ob}}$, i.e., where R, denoted then by $\mathbf{r}$, is very small. In this case, in Equation (14), $\mathbf{r} \wedge[(\mathbf{r}-\mathbf{V r} / \mathrm{c}) \wedge \dot{\mathbf{V}}] / \mathrm{c}^{2}$ is negligible in front of $(\mathbf{r}-\mathbf{V r} / \mathrm{c}) / \beta^{2}$, and $\mathbf{r} \cdot \mathbf{V} / \mathrm{c}$ in front of $\mathrm{r}$. Therefore, at $\mathbf{r}_{\mathrm{ob}}$, the expression for $\mathbf{H}$ denoted then $\mathbf{H}_{\mathrm{ob}}$ is the following

$$
\mathbf{H}_{\mathrm{ob}}=\frac{\mathrm{e}}{4 \pi \mathrm{r}^{3}} \mathbf{V} \wedge \mathbf{r} .
$$

Now $e /\left(4 \pi r^{3}\right)$ is a density of electrical charge that we denote by $\rho_{e}$, i.e., (15) can be written then

$$
\mathbf{H}_{\mathrm{ob}}=\rho_{\mathrm{e}} \mathbf{V} \wedge \mathbf{r} .
$$

Yet, as shown in Equation (3), the magnetic field $\mathbf{H}$ is the velocity $\partial_{t} \xi$ of a point of the ether. Therefore, at $\mathbf{r}_{\mathrm{ob}}$, the expression for $\partial_{t} \xi$ denoted more specifically by $\partial_{t} \xi_{o b}$, is

$$
\partial_{\mathrm{t}} \xi_{\mathrm{ob}}=\mathbf{H}_{\mathrm{ob}} \text {, }
$$

where $\mathbf{H}_{\mathrm{ob}}$ id defined in (15a). It appears therefore, that the velocity $\partial_{\mathrm{t}} \boldsymbol{\xi}_{\mathrm{ob}}$ of a point of the ether at $\mathbf{r}_{\mathrm{ob}}$, defined by (15a) and (16), is of the same form as the velocity $\mathbf{V}_{\Omega}$ of a point on a rotating solid of rotation vector $\boldsymbol{\Omega}$ and of radius $\mathrm{r}$, since $\mathbf{V}_{\Omega}$ is of the form

$$
\mathbf{V}_{\Omega}=\mathbf{\Omega} \wedge \mathbf{r}
$$

One sees, by considering (15a) and (17), that all happens as if

$$
\mathbf{\Omega}=\rho_{\mathrm{e}} \mathbf{V} .
$$

Remark. $\rho_{\mathrm{e}} \mathbf{V}$ has the dimension $[1 / \mathrm{T}]$ like $[\Omega]$, indeed in Zareski (2012), we have shown that in the ether elasticity theory, then $\left[\rho_{\mathrm{e}}\right]=[1 / \mathrm{L}]$.

Now let us take $r=r_{e}$, where $r_{e}$ is the radius of e, in this case $\mathbf{\Omega}$ is the spin of the electron, and will be denoted $\boldsymbol{\Omega}_{\mathrm{e}}$. In a quantum state, e.g., of an hydrogenous atom, one has, Cf. e.g., Equations (10), (43) \& (47) of Zareski (2014), 


$$
\mathrm{V}=\frac{\mathrm{eq}}{4 \pi \varepsilon_{0}} \frac{1}{\mathrm{n} \hbar},
$$

where $\mathrm{q}$ is the positive electric charge of the nucleus considered as immobile around which the electron gravitate. Therefore

$$
\Omega_{\mathrm{e}}= \pm \rho_{\mathrm{e}} \frac{\mathrm{eq}}{4 \pi \varepsilon_{0}} \frac{1}{\mathrm{n} \hbar},
$$

that shows the quantum states of the electron spin in an hydrogenous atom.

\section{Conclusions}

It appears that the particle, of mass $m$ and electrical charge e, is a globule $\hat{\xi}(\mathrm{m}, \mathrm{e})$, i.e., a deformation of the ether that moves with the velocity of the particle. If this particle is an electron, then, in its motion the globule associated to this electron creates, out of it, a field $\xi_{\mathrm{ob}}$ of the displacements of the points of the ether such that near to this globule, the velocities $\partial_{t} \xi_{o b}$ of the points of the ether are of the same form as the velocities of the points of a rotating solid. In a quantum state of an atom, this spin can take only quantized values. These results regarding the elastic interpretation of the electron spin and the results described in Refs. 3,4,5,8 ensue from the "Ether Elasticity Theory" that takes into account Einstein's "General Theory of Relativity".

\section{References}

Einstein, A. (1920). Ether and the theory of relativity. The collected papers of Albert Einstein. Berlin: Springer.

Einstein, A. (1991). Sci. Am (Special Issue 41). Reprinted from the April 1950 issue.

Landau, L. D., \& Lifshitz, E. M. (1962). The classical theory of field. Pergamon Press.

Maxwell, J. C. (1954). A Treatise on Electricity and Magnetism (Dover, New-York, 1954).

Zareski, D. (2001). Found. Phys. Let., 14(5), 447. http://dx.doi.org/10.1023/A:1015513504377

Zareski, D. (2012). Phys. Essays, 25, 2. http://dx.doi.org/10.4006/0836-1398-25.2.268

Zareski, D. (2013). Phys. Essays, 26, 2. http://dx.doi.org/10.4006/0836-1398-26.1.2

Zareski, D. (2014). The Quantum Mechanics as Also a Case of the Ether Elasticity Theory. Applied Physics Research, 6(4), 48-55. http://dx.doi.org/10.5539/apr.v6n4p48

\section{Copyrights}

Copyright for this article is retained by the author(s), with first publication rights granted to the journal.

This is an open-access article distributed under the terms and conditions of the Creative Commons Attribution license (http://creativecommons.org/licenses/by/3.0/). 